\title{
A focus on POCUS
}

\section{An interview with Dr Rob Arntfield}

\author{
Alice Yi, Dino D'Andrea \\ Faculty Reviewer: Robert Arntfield, MD, FRCPC, FCCP, FACEP (Division of Critical Care Medicine)
}

\section{INTRODUCTION}

Dr Rob Arntfield is a trained emergency physician, intensivist, and traumatologist here at London Health Sciences Centre (LHSC). Passionate about the use of ultrasonography and teaching, Dr Arntfield co-authored Point of Care Ultrasound (POCUS), which was published in 2014. A self-described London "townie", he attended Western for medical school and continued in his hometown for a double emergency medicine and intensive care residency before pursuing a fellowship in New York City. We sat down with Dr Arntfield to discuss his work in critical care, trauma, and the rise of POCUS in trauma medicine.

UWOMJ: Can you tell us about your educational background and what drew you in to critical care medicine?

Dr Rob Arntfield: I did my residency training in emergency medicine with the intent of pursuing critical care from that base specialty; I did my critical care fellowship with my emergency training, so that took six years instead of seven years. Along the way I became really cynical of making decisions based off stethoscope or palpation findings and became enamored with the diagnostic power that ultrasound offered at the bedside. The best way to get dedicated ultrasound training in 2010 was to do an ultrasound fellowship. I spent a year at New York City's Mount Sinai for this training before coming back to London to lead an ultrasound program in the intensive care unit.

In terms of being drawn to critical care, mentorship was one of the strongest influences; there were (and are) some incredible emergency physicians in London, and one in particular at the time who pioneered the emergency medicine/critical care pathway locally: Dr. Scott Anderson. He is the original version of what is now known as a "resuscitationist"-someone who dwells in resuscitation, moving from the trauma bay to the emergency room to the intensive care unit. The priorities are similar in each of these environments: restoring circulation, providing oxygenation, airway management, and the synthesis of complex situations. These features that he modeled as well as the balance of intensity and cognitive load was additionally appealing to me.

\section{You've touched on the ability to use POCUS to make diagnoses at the bedside. Could you expand on the value of POCUS and medical sonography in the trauma setting?}

Trauma is fascinating because it affects young people; it's so sudden and often complex. You rapidly need to identify life threatening issues that can be occult to the physical exam. A major concern with trauma is torso hemorrhage or intraperitoneal hemorrhage. Up until the early 90 s, if you came in the trauma bay, the way to diagnose internal bleeding would be to have a surgeon at the bedside in the trauma bay and perform a diagnostic peritoneal lavage (DPL) - they make a small incision your abdomen and see if they can draw out free blood-to determine if you needed to be taken to the OR. So that was the point-of-care test-du-jour. That required a surgeon present at all times, which is pretty demanding on the system. Fast forward to the early 90 s and the advent of the focused assessment with sonography for trauma (FAST exam) - the poster child for POCUS, where you can rule in or rule out hemorrhage noninvasively, and rapidly, by putting the ultrasound probe to three parts of the abdomen.

After the FAST was here to stay it was asked: "What else can we do?" We found that we could rapidly rule in or rule out a pneumothorax, hemothorax, or hemopericardium (when these are all done together with the FAST exam-it is called the "eFAST" exam). There are even more advanced applications that some of us are doing (transesophageal echo or transcranial Doppler) in trauma, but the bedrock of trauma ultrasound still remains the eFAST exam.

$$
\begin{array}{r}
\text { I TRY TO STAY SHY OF BEING A ZEALOT, } \\
\text { BECAUSE JUST AS ULTRASOUND } \\
\text { IS DISRUPTING PHYSICAL EXAM, } \\
\text { SOME NEWER TECHNOLOGY WILL } \\
\text { EVENTUALLY COME AND DISRUPT } \\
\text { ULTRASOUND, BUT FOR NOW, THIS } \\
\text { IS A VERY POWERFUL TOOL THAT WE } \\
\text { CAN WIELD SAFELY, AND STUDENTS } \\
\text { AND PHYSICIANS CAN LEARN EASILY. }
\end{array}
$$

A recent study came out talking about the variability in POCUS training ${ }^{2}-$ do you have any comments regarding there being no certification process?

That study is made up of both emergency physicians and trauma surgeons, so right there, there's the fracture line. Emergency physicians, when they adopted POCUS, did it in a very organized fashion. They formed a society-the Canadian Emergency Ultrasound Society (CEUS). They have a certification pathway-you have to be certified in ultrasound before you can do it in an emergency department, and the CEUS ordains you as being certified once you've done 50 observed FAST exams, for example.

Surgeons, on the other hand, do not have a standardized training pathway. They know the abdomen very well, and their training is a little bit more haphazard. As surgeons are not "certified" in 
appendectomies or caesarean sections, suggesting certification for an ultrasound procedure like the FAST exam would be a difficult precedent.

This brings up the broader certification topic which is very commonly asked of me for ultrasound. Do I need to be certified to read an EKG? Many of us argue that with fundamental applications of ultrasound, certification is not desirable. It should be built in to training the same way that any other skill is. Having training standards is critical, but certification takes a skill and puts it on a pedestal which creates access problems.

\section{What is your role in POCUS teaching and training?}

The biggest thing I do is run a program in the ICU for residents and fellows. In emergency medicine, residents do a month-long rotation. Training for critical care is similar, where we give an initial bolus of course load of technical training, then they get the practical experience. People come and spend a month under my or my fellows' supervision learning ultrasound and getting feedback. One of the big things LHSC does better than anywhere else is that all our machines are wirelessly connected, and so they all save a "movie" of your ultrasound, so if there's any doubt, if you need education, if there's danger, or quality issues, there's recourse. We have nearly 100000 ultrasound studies, which makes us one of the biggest users of POCUS in possibly any hospital in North America. We provide oversight and quality assurance on a lot of the studies, allowing unique opportunities for recourse and education for learners and skilled practitioners alike. This is different to physical exam where unless directly supervised, follow-up and review is not possible. I've now since started a fellowship in ultrasound as well-sort of what I did in the United States for people who not only want to learn how to do ultrasound, they want to learn how to create programs, and to get to an elevated teaching level.

I also do a lot of speaking, nationally and internationally, on the topic, so I spend some time traveling, and trying to push the medium forward using the traditional academic pathways like books and articles, and also through things like Twitter and our own website, screencasts, and education content. So it's kind of a multi-modal, multi-pronged approach, trying to evangelize the technology. I try to stay shy of being a zealot, because just as ultrasound is disrupting physical exam, some newer technology will eventually come and disrupt ultrasound; but for now, this is a very powerful tool that we can wield safely, and students and physicians can learn easily.

\section{Where do you think the field of ultrasonography in trauma is headed in the future?}

We've actually cornered the market really well on the broad brushstrokes and the important high priorities in trauma. The big question in trauma is always, "Where is the bleeding?" We can look for bleeding in the abdomen, in the heart, and in the chest. We can look for pneumothoraces. I do think that ultrasound for guiding procedures in trauma is evolving, for example putting central venous lines in, or doing something called the Resuscitative Endovascular Balloon Occlusion of the Aorta (REBOA), which is new and exciting. I think progress would involve slightly more improved training standards for non-emergency medicine specialties, making sure that every single trauma bay and every single person in every emergency department has those skills would also be part of it.

Things like remote tele-supervision for people in remote areas who don't know how to do the FAST exam have been explored. There was a paper where astronauts were taught how to do a FAST exam on the international space station, ${ }^{3}$ so the idea of tele-mentoring is all very exciting-to be able to guide someone on an expedition, or cruise ship, or wherever.

There are also ultraportable ultrasounds. They're handheld probes that can connect to your tablet or phone. It's probably not ready for prime-time, both in terms of price and in terms of quality. The ultraportable doesn't really apply in people working in existing emergency departments or intensive care units where they have access to high-fidelity machines, but it's for the rural environment, austere environment, wilderness-or in the pre-hospital environment like ambulances and air ambulances. So that ultra-portability market will find its niche, but it is crippled a little bit with needing methods to provide oversight, and that's the challenge. While I was doing my fellowship I worked with the elite rescue paramedics with the New York City Fire Department and trained them using ultra-portable machines. We trained them in FAST and eFAST applications for disaster scenarios. They found particular value in cardiac assessment for presence or absence of activity. One of the things that is difficult in those mass-casualty scenarios is who is dead, and who is alive; who is rescue, and who is recovery. They were really interested in something stronger than their physical exam, and can be rapidly assessed. Being able to gain access to strong visuals for signs of life seem to be very compelling to that group.

\section{Do you have any advice for medical students who are interest- ed in trauma or critical care and/or ultrasonography?}

To be interested in trauma or critical care, in 2016, is to also be interested in ultrasonography. It's not an optional add-on. You can't be practicing trauma or critical care and not be really good at ultrasound, or at least not be capable of it and be a good trauma provider without it. I think if you're interested in POCUS as a primary tool, you may want to go into the specialties that earliest adopted it, so trauma, emergency medicine, critical care, and anesthesia. If you're a student interested in learning POCUS, there's no rush-you will learn these skills during your residency. If you just can't wait, however, there will be opportunities to apply these skills in some clerkship rotations. Studies actually show students can be better diagnosticians with POCUS than senior clinicians are with their stethoscope-so be gracious with skills once you have them! ${ }^{4}$

\section{FURTHER READING}

1. Soni NJ, Arntfield R, Kory P. Point of care ultrasound. 1st ed. Philadelphia: Elsevier; 2015. 393p.

2. AlEassa EM, Ziesmann MT, Kirkpatrick AW, et al. Point of care ultrasonography use and training among trauma providers across Canada. Can J Surg. 2016;59(1):6-8.

3. Chiao L, Sharipov S, Sargsyan AE, et al. Ocular examination for trauma; clinical ultrasound aboard the International Space Station. J Trauma. 2005;58(5):885-9.

4. Kobal SL, Trento L, Baharami S, et al. Comparison of effectiveness of hand-carried ultrasound to bedside cardiovascular physical examination. Am J Cardiol. 2005 Oct 1;96(7):1002-6. 